\title{
S100B, NSE and MMP-9 fail to predict neurologic outcome while elevated S100B associates with milder initial clinical
}

\section{presentation after aneurysmal subarachnoid hemorrhage}

Heikki Kiiski, MD ${ }^{1}$, Jaakko Långsjö, MD,PhD ${ }^{1}$, Jyrki Tenhunen, MD,PhD ${ }^{1,2}$, Marika Ala-Peijari, MD ${ }^{1}$, Heini Huhtala, $\mathrm{MSc}^{3}$, Mari Hämäläinen, $\mathrm{PhD}^{4}$, Eeva Moilanen, $\mathrm{MD}, \mathrm{PhD}^{4}$, Jukka Peltola, $\mathrm{MD}, \mathrm{PhD}^{5}$

${ }^{1}$ Critical Care Medicine Research Group, Department of Intensive Care, Tampere University Hospital, Tampere, Finland

${ }^{2}$ Department of Surgical Sciences, Division of Anesthesiology and Intensive Care, Uppsala University, Uppsala, Sweden

${ }^{3}$ Faculty of Social Sciences, University of Tampere, Tampere, Finland

${ }^{4}$ The Immunopharmacology Research Group, Faculty of Medicine and Life Sciences, University of Tampere, and Tampere University Hospital, Tampere, Finland

${ }^{5}$ Department of Neurology, University of Tampere and Tampere University Hospital, Tampere, Finland

Corresponding author: Heikki Kiiski, Department of Intensive Care, Tampere University Hospital, PL 2000, TAMPERE. e-mail: heikki.kiiski@fimnet.fi 


\begin{abstract}
Objective: Despite advances in the treatment of aneurysmal subarachnoid hemorrhage (aSAH) one-year mortality remains approximately 50\%. Making an accurate prognosis at the early phase of the disease is notoriously difficult. A clinically reliable biomarker that could be used for better prediction of prognosis and/or as a surrogate for developing complications after aSAH is still lacking. In this study, we evaluated the prognostic values of three promising biomarkers, i.e. S100B, NSE, and MMP-9 in aSAH.
\end{abstract}

Methods: In this prospective population-based study, S100B, NSE, and MMP-9 levels were measured in 47 aSAH patients for up to five days. Blood samples were taken at 0,12 and $24 \mathrm{~h}$ after the admission to the intensive care unit (ICU) and daily after that until the patient was transferred from the ICU. The patients' neurological outcome was evaluated with the modified Rankin Scale $(\mathrm{mRS})$ at six months after aSAH.

Results: Biomarker-levels measured during the first 24 hours were not associated with neurological outcome. S100B levels during the first 24 hours were elevated in patients with a nonsevere initial clinical presentation. Otherwise, there was no association between selected clinical variables and the early biomarker levels. In 22 patients, whose ICU follow-up lasted for up to five days, the total release of biomarkers was not associated with the neurological outcome.

Conclusions: None of the measured biomarkers were associated with the neurological outcome evaluated at six months after aSAH. Elevated levels of S100B in patients with non-severe initial presentation suggest an adaptive role of this biomarker in aSAH. Based on our findings it is not advisable to use these biomarkers to guide clinical decision-making in patients with aSAH. 


\section{Introduction}

Aneurysmal subarachnoid hemorrhage (aSAH) remains a devastating disease with one-year mortality approximately 50\% [1]. Patients suffering aSAH are on average significantly younger than those suffering from other types of stroke with an incidence peak around 50 years [2-4]. aSAH causes significant individual and socioeconomic burden since only one-third of patients gain functional independence and merely $25 \%$ of those report complete recovery without psychosocial or neurological problems [5].

Making an accurate prognosis at the early phase of the disease is notoriously difficult. Pathophysiology of the early brain injury and the secondary brain injury processes after the primary insult are still only partly understood, and timing of the evidence-based therapies is still cumbersome to determine [6-9]. There is a dire need for a clinically reliable biomarker, which could be used for better prediction of prognosis and/or as a surrogate for developing complications after aSAH. Despite extensive research on the topic, a reliable biomarker is still lacking [10-14].

The purpose of this study was to evaluate the prognostic potential, mutual interdependence of biokinetics and association with selected clinical parameters of S100 calcium-binding protein B (S100B), neuron-specific enolase (NSE) and matrix metalloproteinase-9 (MMP-9). S100B was chosen for its' hypothesized ability to reflect glial injury, NSE as a surrogate for neural injury and MMP-9 as a surrogate for blood-brain-barrier (BBB) injury.

S100B is produced mainly by glial cells and it has paracrine and autocrine effects on neural tissue, such as regulating the dynamics of cytoskeleton constituents, protein phosphorylation and cell cycle progression [15][16]. Numerous studies have associated elevated S100B levels and poor outcome in TBI $[17,18]$. However, controversy regarding the prognostic potential of S100B exists. Even 100\% mortality has been reported to associate with high initial S100B levels [19]. Confusingly, only $20 \%$ mortality was observed with similar cut-off values in another study [20]. 
NSE is a neuron-specific form of glycolytic enzyme, enolase. It is located in the cytoplasm of a neuron and seems to contribute to the regulation of chloride concentration during neural activity [21]. It is released passively from neurons when they destruct. Despite the high concentrations of NSE observed in TBI, it has been difficult to show its value in the quantification of brain injury or to differentiate between primary and secondary brain injury [17]. There is also contradictory evidence concerning the prognostic potential of NSE after aSAH [10].

MMP-9 is a member of metzincin family of proteases, which function mainly extracellularly. MMP-9 seems to have a unique role in brain physiology as well as in pathology [22]. In the central nervous system, MMP-9 can be released from various cells, e.g., neurons, glia and leukocytes [22]. In SAH MMP-9 has been associated with the breakdown of BBB due to degradation of tight junction proteins responsible for BBB integrity [12]. Breakage of BBB leads to increased cerebral edema and activation of neuronal and vascular apoptosis [10]. Both blood and cerebrospinal fluid MMP-9 levels have been associated with poor outcome after SAH [23].

In the present study, our aim was to evaluate the prognostic potentials and associations with selected clinical variables of S100, NSE, and MMP-9 after aSAH. 


\section{Methods}

\section{Study design and patients}

Following the approval of the institutional ethics committee, we conducted a prospective, observational, single-center clinical study in Tampere University Hospital (Tampere, Finland). The hospital is one of the five tertiary referral centers in Finland serving a population of approximately 1 million inhabitants and, thus, providing care for all patients suffering from aSAH in the area. The clinical data from this patient cohort has been reported in the previously published study [24]. This study has been registered to ClinicalTrials.gov (NCT02026596).

The study cohort consisted of 61 consecutive aSAH patients admitted to our center from March 2013 to December 2013. Written informed consent was obtained from each patient or from their next of kin. The time of the onset of symptoms associated with aSAH was registered from the patient records. We excluded patients with an unknown time of onset of symptoms as well as patients whose samples for the S100B, NSE, and MMP-9 assays were not collected within the first $24 \mathrm{~h}$ after the onset of symptoms. In total, 47 patients were considered eligible and were, thus, included in the study. All patients were treated according to standardized in-house guidelines.

\section{Clinical evaluation of the patients}

The severity of the initial clinical presentation was evaluated according to the World Federation of Neurological Surgeons (WFNS) grading scale. The extent of the primary hemorrhage on the CT scan was graded with Fisher scale. WFNS was dichotomized into non-severe (WFNS 1-3) and severe (WFNS 4-5). The Fisher grade was dichotomized into non-severe (Fisher 1-2) and severe (Fisher 34).

The neurological outcome was evaluated with the modified Rankin Scale (mRS) six months after aSAH based on a structured interview performed on the telephone or during an outpatient clinic visit. 
mRS was dichotomized into favorable (mRS 0-2) and unfavorable outcome (mRS 3-6). Detailed categorizations of WFNS, Fisher, and mRS are presented in a Supplementary Table.

Acute hydrocephalus was defined as the need for ventriculostomy on a clinical basis during the first 24 hours. Additionally, we assessed the incidence of infection, which was defined as a need for antimicrobial medication during the follow-up period.

In a subgroup of 22 patients who had up to five days' follow-up, we also checked if the patient was treated for delayed cerebral ischemia (DCI). Treatment of DCI was initiated based on clinical evaluation.

\section{Assessment of biomarkers}

Plasma concentrations of S100B, NSE and MMP-9 were measured in the samples collected at 0, 12 and $24 \mathrm{~h}$ after the admission, and after that at every $24 \mathrm{~h}$ for up to five days or until the patient was transferred from the ICU. Before the statistical analysis, the S100B, NSE, and MMP-9 measurements were divided into consecutive $24 \mathrm{~h}$ intervals starting from the onset of symptoms. If S100B, NSE, and MMP-9 were measured more than once per interval, the mean concentration was used in the analysis.

Blood samples were collected into EDTA-containing tubes from an arterial cannula that had been routinely inserted for invasive blood pressure monitoring as well as for blood sampling. After collection, the sample was immediately delivered to the laboratory where it was centrifuged for 10 min at $2000 \mathrm{~g}$ (room temperature). After centrifugation, the plasma was collected and kept at $-70^{\circ} \mathrm{C}$.

S100B, NSE and MMP-9 concentrations in the plasma samples were measured by ELISA with reagents from Cloud-Clone Corp, Houston, TX, USA (S100B and NSE), and from R\&D Systems Europe Ltd, Abingdon, UK (MMP-9). The detection limits and inter-assay coefficient of variations 
were $0.0156 \mu \mathrm{g} / \mathrm{L}$ and $10.2 \%$ for S100B, $0.313 \mu \mathrm{g} / \mathrm{L}$ and $8.4 \%$ for NSE, and $0.0078 \mu \mathrm{g} / \mathrm{L}$ and $7.0 \%$ for MMP-9, respectively.

\section{Statistical analysis}

Statistical analyses were performed with R (version 3.4.1 for Mac OS X). Mann-Whitney U-test was used for continuous variables. Linear regression was used for the time interval analysis. Spearman's correlation was used to estimate whether S100B, NSE and MMP-9 levels had bilateral correlations. To approximate the total release of a biomarker, area under the curve was estimated by trapezoidal method. 


\section{Results}

The basic characteristics of the study cohort are presented in TABLE 1 . These chacteristics have been previously published by our group [24].

Biomarkers levels during the first 24 hours (DAY 1) after aSAH were not associated with the severity of initial CT findings, development of acute hydrocephalus or infection during follow-up. Also, none of the measured biomarkers were associated with patients' neurological outcome evaluated at six months after aSAH. Interestingly, elevated S100B levels during DAY 1 were strongly associated with non-severe initial clinical representation measured with the WFNS scale (FIGURE 1). This was not observed with NSE or MMP-9. Biomarker levels at DAY 1 did not differ between genders and were not affected by age. Statistics for S100B, NSE, and MMP-9 at DAY 1 are listed in TABLES 2-4, respectively.

DAY 1 levels of S100B and NSE were not correlated. However, they were significantly correlated at DAY 2 after which there were significant correlations also at DAY 4 and DAY 5. (TABLE 5). Table 5 also shows the decline of participants during follow-up. MMP-9 was not associated with S100B or NSE levels at any timepoint during the five day ICU follow-up, data not shown.

The subgroup of patients whose ICU follow-up lasted for up to five days $(n=22)$ was assessed for biomarker level changes using linear regression. None of the studied biomarkers showed a statistically significant change in time when analyzed for the whole subgroup or when subgroup was further divided based on the severity of clinical presentation, initial CT findings or outcome (TABLE 6). The total release of biomarkers during the five-day ICU follow-up in this subgroup was not associated with the neurological outcome for any of the biomarkers (TABLE 7). Total release of NSE was marginally higher in patients without DCI $(\mathrm{p}=0.049)$. Otherwise there were no significant correlations with DCI and biomarker levels (TABLE 7). Also, in this subgroup at the end of five-day ICU follow-up (DAY 5) biomarker levels were not associated with the neurological outcome 
(TABLE 8). The chosen treatment method was not associated with the biomarker levels at any timepoint (TABLE 9). In a subgroup of patients with up to five days ICU follow-up the total release of biomarkers were calculated with an area under the curve by using the trapezoidal method. Total release of biomarkers was not associated with the selected treatment method (TABLE 10). Two patients died before the aneurysm was secured. In two patients, treatment of the aneurysm was not technically feasible during the follow-up. These four patients were excluded from the analysis of associations between biomarker levels and treatment modality. 


\section{Discussion}

In our patient cohort S100B, NSE and MMP-9 levels were not associated with the neurological outcome measured at six months after aSAH by using modified Ranking Scale. On the other hand, elevated S100B, but not NSE or MMP-9, at DAY 1 was strongly associated with non-severe initial clinical representation suggesting a possible adaptive role of S100B in aSAH.

Findings regarding the prognostic potential of S100B, NSE and MMP-9 after different types of brain injury have been inconsistent $[10,17]$. For S100B and NSE, our findings are in line with previous observations by Olivecrona et al. [25] who found no significant value of these markers as predictors of clinical outcome in patients suffering from severe TBI. Also, Piazza et al. [26] observed in pediatric TBI patients that S100B was elevated but did not correlate with neurological outcome.

Quite the opposite results have been documented recently in aSAH patients. One study reported $100 \%$ mortality with S100B values $>0.7 \mu \mathrm{g} / \mathrm{L}$ after aSAH [19]. In another study, early S100B and NSE levels were found to predict the neurological outcome with $100 \%$ specificity in poor-grade aSAH patients [27]. Moritz et al. studied serum and cerebrospinal fluid (CSF) levels of S100B and NSE in aSAH patients during ICU stay. They found that elevated S100B levels in serum and CSF and NSE levels in CSF were associated with neurological outcome while NSE levels in serum had no predictive value [28]. Quintard et al. found that elevated S100B levels in the peripheral blood during the first week after aSAH were associated with poor neurological outcome. Predictive value was strongest for S100B levels at DAY 5. They also found that NSE levels from DAY 5 to DAY 7 were associated with poor neurological outcome, while earlier NSE levels were not [29].

Kellerman et al. [19] suggest that S100B levels might be used to guide clinical decision-making. However, reported cut-off values for $100 \%$ mortality and for poor neurological outcome have varied significantly in the literature [20,28]. Also, there has been uncertainty whether a single time-point measurement or the total release of S100B during the early phase of the disease should be used in 
prognostication [20]. One recent retrospective study observed an association between elevated NSE levels and poor functional outcome after aSAH. They used only the first NSE measurement for each patient in the analysis. Timepoints in which measurements were made varied between patients making it difficult to conclude the temporal profile of NSE levels [30].

In our patient cohort the early levels of the biomarkers and the their total release during the fiveday ICU follow-up were both unable to predict the neurological outcome. In fact, in our cohort those presenting less severe initial clinical symptoms had a propensity to significantly higher S100B values (median $0.741 \mu \mathrm{g} / \mathrm{L}$ ), thus, strongly encouraging to caution in prognostication based on S100B. Based on our findings in patients with aSAH and the previous data showing no prognostic value of S100B or NSE in TBI patients [25], we are prone to take a cautious stand in clinical decision making based on S100B and NSE during the first five days after aSAH.

It seems that dualistic view of elevated S100B concentration as "good" or "bad" is not biologically appropriate [31]. In experimental models of neural cells S100B has shown neuroprotective properties, e.g., after glucose deprivation there was less cell death and mitochondrial dysfunction in cells exposed to S100B [32]. It has also been well documented that in neural cultures the effects of S100B depend on the concentration. Concentrations up to nanomolar level seem to be neurotrophic while micromolar concentrations were shown to be apoptotic and toxic [33,34]. Most assays used for S100B detect up to $20-25 \mu \mathrm{g} / \mathrm{L}$ which is in the nanomolar scale [31]. It is possible that in these concentrations elevation in S100B reflects an adaptive response to the neural stress. In our patient cohort we observed high levels of S100B in patients with a non-severe initial clinical presentation. This suggests that secretion of S100B from glial cells might be an adaptive response to the brain injury since high S100B levels were not associated with neurological outcome. Similar conclusions have been drawn about the observation from electroconvulsive therapy study where patients with elevated S100B levels had the best response to treatment [35]. 
One study has been able to show an association of elevated peripheral blood MMP-9 levels and vasospasm in aSAH patients [36]. In contrast, Chou et al. found that elevated blood and CSF MMP9 levels correlated with poor neurological outcome at three months after aSAH [23] but no association to vasospasm was detected. In our study MMP-9 levels were not associated with the neurological outcome nor development of DCI.

In a subgroup of patients ( $\mathrm{n}=22$ ) with five-day ICU follow-up there was no association between biomarker levels and neurological outcome at DAY 5. In that subgroup those who were not clinically diagnosed with DCI had statistically higher NSE levels. Due to the small sample size and relatively weak statistical significance it does not allow definite conclusions to be drawn about the role of NSE in DCI. Additionally, the selected treatment method was not associated with the biomarker levels.

It is well known that the typically used outcome scales (i.e. mRS and Glasgow Outcome Scale) after aSAH are not sensitive for more subtle impairments in the executive functions which may cause a significant reduction in the quality of life after aSAH [37,38]. Based on our findings it is not possible to evaluate whether elevation in these biomarkers is associated with brain injury causing these more subtle deficits. More sophisticated methods might be advantageous to detect such impairments [37,39]. Despite the relatively small sample size, our findings raise a significant doubt against the clinical utility of S100B, NSE, and MMP-9 as prognostic biomarkers after aSAH.

\section{Conclusions}

None of the measured plasmatic biomarkers were associated with the neurological outcome evaluated at 6 months after aSAH in the present patient population. S100B levels were higher in patients with non-severe initial clinical presentation suggesting that S100B might have an adaptive role in the brain injury after aSAH since the elevated levels were not associated with the neurological outcome. Based on our findings it is not advisable to use these plasmatic biomarkers to guide clinical decision-making in patients with aSAH. 


\section{Author contributions}

HK contributed to the study conception, neurological outcome evaluations, statistical analyses, and drafted the manuscript. JL contributed to the study conception, patient recruitment, and drafted the manuscript. JT contributed to the study conception, MA-P contributed to patient recruitment, evaluation of the initial clinical severity, and evaluation of the initial computed tomography findings. $\mathrm{HH}$ contributed to the statistical analyses. $\mathrm{MH}$ contributed to the laboratory analysis of the biomarkers. EM contributed to the laboratory analysis of the biomarkers. JP contributed to the study conception, statistical analyses, neurological outcome evaluations, and drafted the manuscript. All authors revised the manuscript for intellectual content and approved the final version for submission. 


\section{Conflicts of interest}

Dr. Långsjö has received lecture compensation from Orion Pharma and holds small amount of Orion Pharma stock. Dr. Långsjö has attented an educational congress as a guest of Pfizer Pharmaceuticals.

Jukka Peltola has participated in clinical trials for Eisai, UCB, and Bial; received research grants from Eisai, Medtronic, UCB, and Cyberonics; received speaker honoraria from Cyberonics, Eisai, Medtronic, Orion Pharma, Sandoz and UCB; received support for travel congresses from Cyberonics, Eisai, Medtronic Sandoz, and UCB; and participated in advisory boards for Cyberonics, Eisai, Medtronic, UCB and Pfizer.

Other authors declare that the research was conducted in the absence of any commercial or financial relationships that could be construed as a potential conflict of interest. 


\section{Funding}

The study was financially supported by Academy of Finland (Grant number 138402) and the Competitive State Research Financing of the Expert Responsibility area of Tampere University Hospital. 


\section{Acknowledgements}

Ms. Meiju Kukkonen and Terhi Salonen are acknowledged for their excellent technical assistance. 


\section{References}

[1] M. Korja, K. Silventoinen, T. Laatikainen, P. Jousilahti, V. Salomaa, J. Kaprio, Causespecific mortality of 1-year survivors of subarachnoid hemorrhage, Neurology. 80 (2013) 481-486. doi:10.1212/WNL.0b013e31827f0fb5.

[2] M.T. Lawton, G.E. Vates, Subarachnoid Hemorrhage, N Engl J Med. 377 (2017) 257-266. doi:10.1056/NEJMcp1605827.

[3] B.E. Zacharia, Z.L. Hickman, B.T. Grobelny, P. DeRosa, I. Kotchetkov, A.F. Ducruet, et al., Epidemiology of Aneurysmal Subarachnoid Hemorrhage, Neurosurgery Clinics of North America. 21 (2010) 221-233. doi:10.1016/j.nec.2009.10.002.

[4] B.M. Kissela, J.C. Khoury, K. Alwell, C.J. Moomaw, D. Woo, O. Adeoye, et al., Age at stroke: temporal trends in stroke incidence in a large, biracial population, Neurology. 79 (2012) 1781-1787. doi:10.1212/WNL.0b013e318270401d.

[5] M.J.H. Wermer, H. Kool, K.W. Albrecht, G.J.E. Rinkel, Aneurysm Screening after Treatment for Ruptured Aneurysms Study Group, Subarachnoid hemorrhage treated with clipping: long-term effects on employment, relationships, personality, and mood, Neurosurgery. 60 (2007) 91-7- discussion 97-8. doi:10.1227/01.NEU.0000249215.19591.86.

[6] R.L. Macdonald, Delayed neurological deterioration after subarachnoid haemorrhage, Nat Rev Neurol. 10 (2013) 44-58. doi:10.1038/nrneurol.2013.246.

[7] M.J. Rowland, G. Hadjipavlou, M. Kelly, J. Westbrook, K.T.S. Pattinson, Delayed cerebral ischaemia after subarachnoid haemorrhage: looking beyond vasospasm, British Journal of Anaesthesia. 109 (2012) 315-329. doi:10.1093/bja/aes264.

[8] R.L. Macdonald, M.N. Diringer, G. Citerio, Understanding the disease: aneurysmal subarachnoid hemorrhage, Intensive Care Med. 40 (2014) 1940-1943. doi:10.1007/s00134014-3483-5.

[9] C.L. Francoeur, S.A. Mayer, Management of delayed cerebral ischemia after subarachnoid hemorrhage, Crit Care. 20 (2016) 137-12. doi:10.1186/s13054-016-1447-6.

[10] C.M. Hong, C. Tosun, D.B. Kurland, V. Gerzanich, D. Schreibman, J.M. Simard, Biomarkers as outcome predictors in subarachnoid hemorrhage - a systematic review, Biomarkers. 19 (2014) 95-108. doi:10.3109/1354750X.2014.881418.

[11] M.M. Przybycien-Szymanska, W.W. Ashley Jr., Biomarker Discovery in Cerebral Vasospasm after Aneurysmal Subarachnoid Hemorrhage, Journal of Stroke and Cerebrovascular Diseases. 24 (2015) 1453-1464. doi:10.1016/j.jstrokecerebrovasdis.2015.03.047.

[12] B. Lucke-Wold, A. Logsdon, B. Manoranjan, R. Turner, E. McConnell, G. Vates, et al., Aneurysmal Subarachnoid Hemorrhage and Neuroinflammation: A Comprehensive Review, Ijms. 17 (2016) 497-17. doi:10.3390/ijms 17040497.

[13] H. Kiiski, J. Långsjö, J. Tenhunen, M. Ala-Peijari, H. Huhtala, M. Hämäläinen, et al., Time-courses of plasma IL-6 and HMGB-1 reflect initial severity of clinical presentation but do not predict poor neurologic outcome following subarachnoid hemorrhage, eNeurologicalSci. 6 (2017) 55-62. doi:10.1016/j.ensci.2016.11.010.

[14] H. Kiiski, V. Jalkanen, M. Ala-Peijari, M. Hämäläinen, E. Moilanen, J. Peltola, et al., Plasma Soluble Urokinase-Type Plasminogen Activator Receptor Is Not Associated with Neurological Outcome in Patients with Aneurysmal Subarachnoid Hemorrhage, Front. Neurol. 8 (2017) 8-7. doi:10.3389/fneur.2017.00144.

[15] C. Adami, G. Sorci, E. Blasi, A.L. Agneletti, F. Bistoni, R. Donato, S100B expression in and effects on microglia, Glia. 33 (2001) 131-142.

[16] R. Donato, Intracellular and extracellular roles of S100 proteins, Microsc. Res. Tech. 60 
(2003) 540-551. doi:10.1002/jemt.10296.

[17] S. Mrozek, J. Dumurgier, G. Citerio, A. Mebazaa, T. Geeraerts, Biomarkers and acute brain injuries: interest and limits, Crit Care. 18 (2014) 220. doi:10.1186/cc13841.

[18] S. Giacoppo, P. Bramanti, M. Barresi, D. Celi, V. Foti Cuzzola, E. Palella, et al., Predictive Biomarkers of Recovery in Traumatic Brain Injury, Neurocrit Care. 16 (2012) 470-477. doi:10.1007/s12028-012-9707-z.

[19] I. Kellermann, A. Kleindienst, N. Hore, M. Buchfelder, S. Brandner, Early CSF and Serum S100B Concentrations for Outcome Prediction in Traumatic Brain Injury and Subarachnoid Hemorrhage, Clinical Neurology and Neurosurgery. 145 (2016) 79-83. doi:10.1016/j.clineuro.2016.04.005.

[20] M. Olivecrona, L.-O.D. Koskinen, Comment on: Early CSF and serum S 100B concentrations for outcome prediction in traumatic brain injury and subarachoid haemorrhage, Clinical Neurology and Neurosurgery. 150 (2016) 197-198. doi:10.1016/j.clineuro.2016.07.026.

[21] P.J. Marangos, D.E. Schmechel, Neuron specific enolase, a clinically useful marker for neurons and neuroendocrine cells, Annu. Rev. Neurosci. 10 (1987) 269-295. doi:10.1146/annurev.ne.10.030187.001413.

[22] B. Vafadari, A. Salamian, L. Kaczmarek, MMP-9 in translation: from molecule to brain physiology, pathology, and therapy, Journal of Neurochemistry. 139 (2016) 91-114. doi:10.1111/jnc. 13415.

[23] S.H.-Y. Chou, S.K. Feske, S.L. Simmons, R.G.J. Konigsberg, S.C. Orzell, A. Marckmann, et al., Elevated Peripheral Neutrophils and Matrix Metalloproteinase 9 as Biomarkers of Functional Outcome Following Subarachnoid Hemorrhage, Transl. Stroke Res. 2 (2011) 600-607. doi:10.1007/s12975-011-0117-x.

[24] H. Kiiski, J. Tenhunen, M. Ala-Peijari, H. Huhtala, M. Hämäläinen, J. Långsjö, et al., Increased plasma UCH-L1 after aneurysmal subarachnoid hemorrhage is associated with unfavorable neurological outcome, Journal of the Neurological Sciences. 361 (2016) 144149. doi:10.1016/j.jns.2015.12.046.

[25] M. Olivecrona, M. Rodling-Wahlstrom, S. Naredi, L.-O.D. Koskinen, S-100B and neuron specific enolase are poor outcome predictors in severe traumatic brain injury treated by an intracranial pressure targeted therapy, Journal of Neurology, Neurosurgery \& Psychiatry. 80 (2009) 1241-1247. doi:10.1136/jnnp.2008.158196.

[26] O. Piazza, M.P. Storti, S. Cotena, F. Stoppa, D. Perrotta, G. Esposito, et al., S100B is not a reliable prognostic index in paediatric TBI, Pediatr Neurosurg. 43 (2007) 258-264. doi: $10.1159 / 000103304$.

[27] T. Abboud, K.C. Mende, R. Jung, P. Czorlich, E. Vettorazzi, M. Priefler, et al., Prognostic Value of Early S100 Calcium Binding Protein B and Neuron-Specific Enolase in Patients with Poor-Grade Aneurysmal Subarachnoid Hemorrhage: A Pilot Study, World Neurosurgery. 108 (2017) 669-675. doi:10.1016/j.wneu.2017.09.074.

[28] S. Moritz, J. Warnat, S. Bele, B.M. Graf, C. Woertgen, The prognostic value of NSE and S100B from serum and cerebrospinal fluid in patients with spontaneous subarachnoid hemorrhage, J Neurosurg Anesthesiol. 22 (2010) 21-31. doi:10.1097/ANA.0b013e3181bdf50d.

[29] H. Quintard, S. Leduc, P. Ferrari, I. Petit, C. Ichai, Early and persistent high level of PS $100 \beta$ is associated with increased poor neurological outcome in patients with $\mathrm{SAH}$ : is there a PS $100 \beta$ threshold for SAH prognosis? Crit Care. 20 (2016) 1711-9. doi:10.1186/s13054016-1200-1.

[30] R.G. Tawk, S.S. Grewal, M.G. Heckman, B. Rawal, D.A. Miller, D. Edmonston, et al., The Relationship Between Serum Neuron-Specific Enolase Levels and Severity of Bleeding and Functional Outcomes in Patients With Nontraumatic Subarachnoid Hemorrhage, 
Neurosurgery. 78 (2016) 487-491. doi:10.1227/NEU.0000000000001140.

[31] C.-A. Gonçalves, M. Concli Leite, P. Nardin, Biological and methodological features of the measurement of S100B, a putative marker of brain injury, Clinical Biochemistry. 41 (2008) 755-763. doi:10.1016/j.clinbiochem.2008.04.003.

[32] S.W. Barger, L.J. Van Eldik, M.P. Mattson, S100 beta protects hippocampal neurons from damage induced by glucose deprivation, Brain Res. 677 (1995) 167-170.

[33] R. Donato, S100: a multigenic family of calcium-modulated proteins of the EF-hand type with intracellular and extracellular functional roles, Int. J. Biochem. Cell Biol. 33 (2001) 637-668.

[34] R. Donato, G. Sorci, F. Riuzzi, C. Arcuri, R. Bianchi, F. Brozzi, et al., S100B's double life: Intracellular regulator and extracellular signal, BBA - Molecular Cell Research. 1793 (2009) 1008-1022. doi:10.1016/j.bbamcr.2008.11.009.

[35] J. Palmio, M. Huuhka, S. Laine, H. Huhtala, J. Peltola, E. Leinonen, et al., Electroconvulsive therapy and biomarkers of neuronal injury and plasticity: Serum levels of neuron-specific enolase and S-100b protein, Psychiatry Research. 177 (2010) 97-100. doi:10.1016/j.psychres.2009.01.027.

[36] M.J. McGirt, J.R. Lynch, R. Blessing, D.S. Warner, A.H. Friedman, D.T. Laskowitz, Serum von Willebrand factor, matrix metalloproteinase-9, and vascular endothelial growth factor levels predict the onset of cerebral vasospasm after aneurysmal subarachnoid hemorrhage, Neurosurgery. 51 (2002) 1128-34- discussion 1134-5.

[37] E.S. Connolly, A.A. Rabinstein, J.R. Carhuapoma, C.P. Derdeyn, J. Dion, R.T. Higashida, et al., Guidelines for the Management of Aneurysmal Subarachnoid Hemorrhage: A Guideline for Healthcare Professionals From the American Heart Association/American Stroke Association, Stroke. 43 (2012) 1711-1737. doi:10.1161/STR.0b013e3182587839.

[38] T. Al-Khindi, R.L. Macdonald, T.A. Schweizer, Cognitive and functional outcome after aneurysmal subarachnoid hemorrhage, Stroke. 41 (2010) e519-36. doi:10.1161/STROKEAHA.110.581975.

[39] S. Wallmark, E. Lundström, J. Wikström, E. Ronne-Engström, Attention deficits after aneurysmal subarachnoid hemorrhage measured using the test of variables of attention, Stroke. 46 (2015) 1374-1376. doi:10.1161/STROKEAHA.115.009092. 


\section{Figure and Figure Legend}

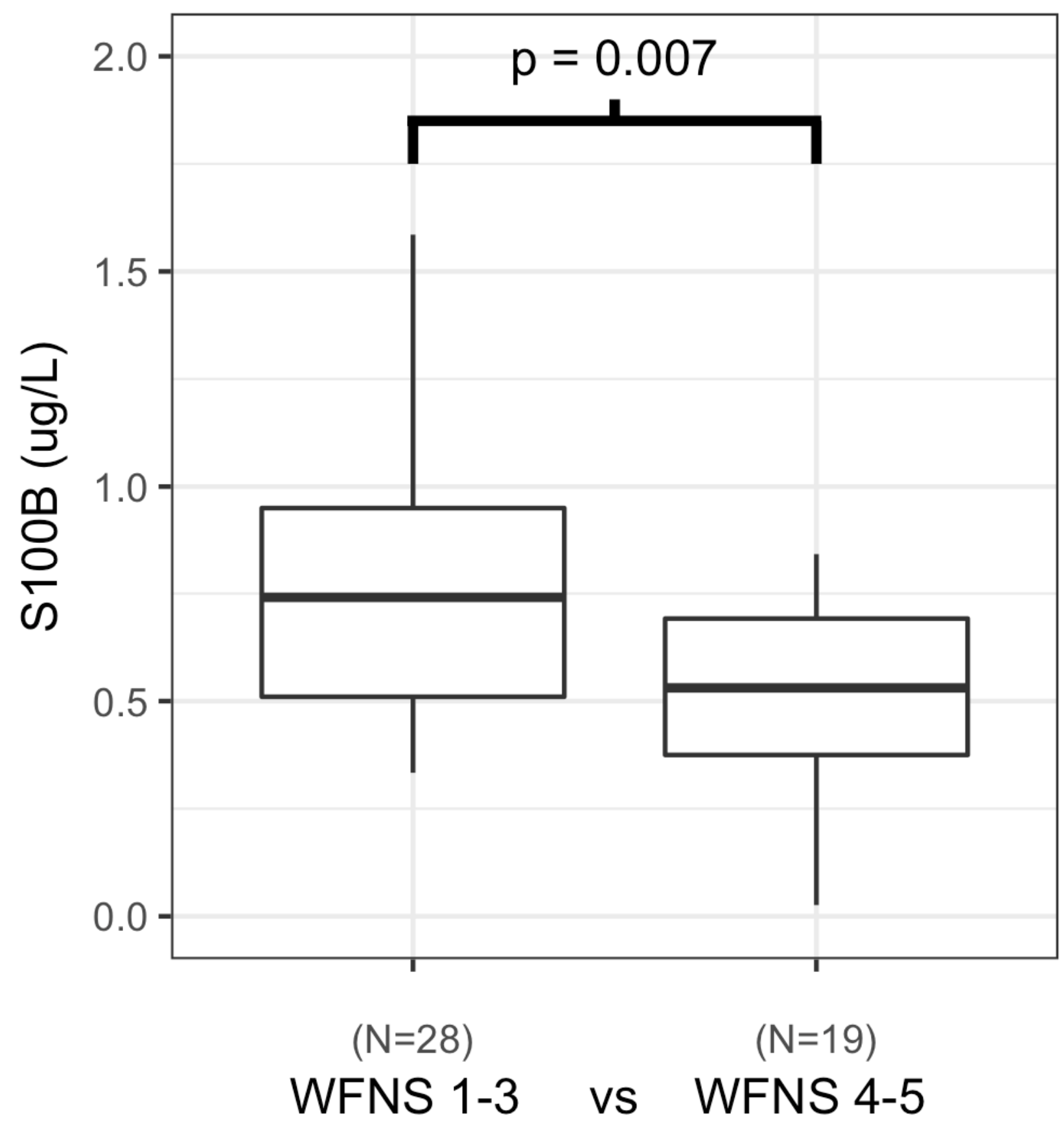

Figure 1. S100B levels measured within the first 24 hours after aSAH in relation to the severity of initial clinical presentation (WFNS). Patients with non-severe clinical presentations had significantly higher S100B levels $(\mathrm{p}=0.007)$. Outliers not shown. 